Revue des patrimoines

$31 \mid 2017$

Patrimoines de la santé : essais de définition - enjeux de conservation

\title{
Le CHU Dijon Bourgogne : une politique active de conservation des collections scientifiques et techniques
}

The university hospital of Dijon, in Burgundy, an active policy of preservation of scientific and technical collections

\section{Adeline Rivière}

\section{OpenEdition}

Journals

\section{Édition électronique}

URL : http://journals.openedition.org/insitu/14165

DOI : 10.4000/insitu.14165

ISSN : 1630-7305

\section{Éditeur}

Ministère de la Culture

\section{Référence électronique}

Adeline Rivière, «Le CHU Dijon Bourgogne : une politique active de conservation des collections scientifiques et techniques », In Situ [En ligne], 31 | 2017, mis en ligne le 24 février 2017, consulté le 09 octobre 2020. URL : http://journals.openedition.org/insitu/14165 ; DOI : https://doi.org/10.4000/ insitu. 14165

Ce document a été généré automatiquement le 9 octobre 2020.

\section{c) (†) $\Theta$}

In Situ Revues des patrimoines est mis à disposition selon les termes de la licence Creative Commons Attribution - Pas d'Utilisation Commerciale - Pas de Modification 4.0 International. 


\section{Le CHU Dijon Bourgogne : une} politique active de conservation des collections scientifiques et techniques

The university hospital of Dijon, in Burgundy, an active policy of preservation of scientific and technical collections

Adeline Rivière

1 Actuellement, le centre hospitalier universitaire (CHU) Dijon Bourgogne emploie plus de 7200 salariés répartis dans 15 pôles de spécialités médicales et médico-techniques et 15 directions. L'établissement dispose d'une capacité d'accueil de 1777 lits/places; 5000 repas sont préparés chaque jour pour les patients et le personnel. En somme, il s'agit d'une véritable ville au service des patients de toute la région et au-delà, avec une place très importante octroyée aux missions de recherche médicale. 
Figure 1

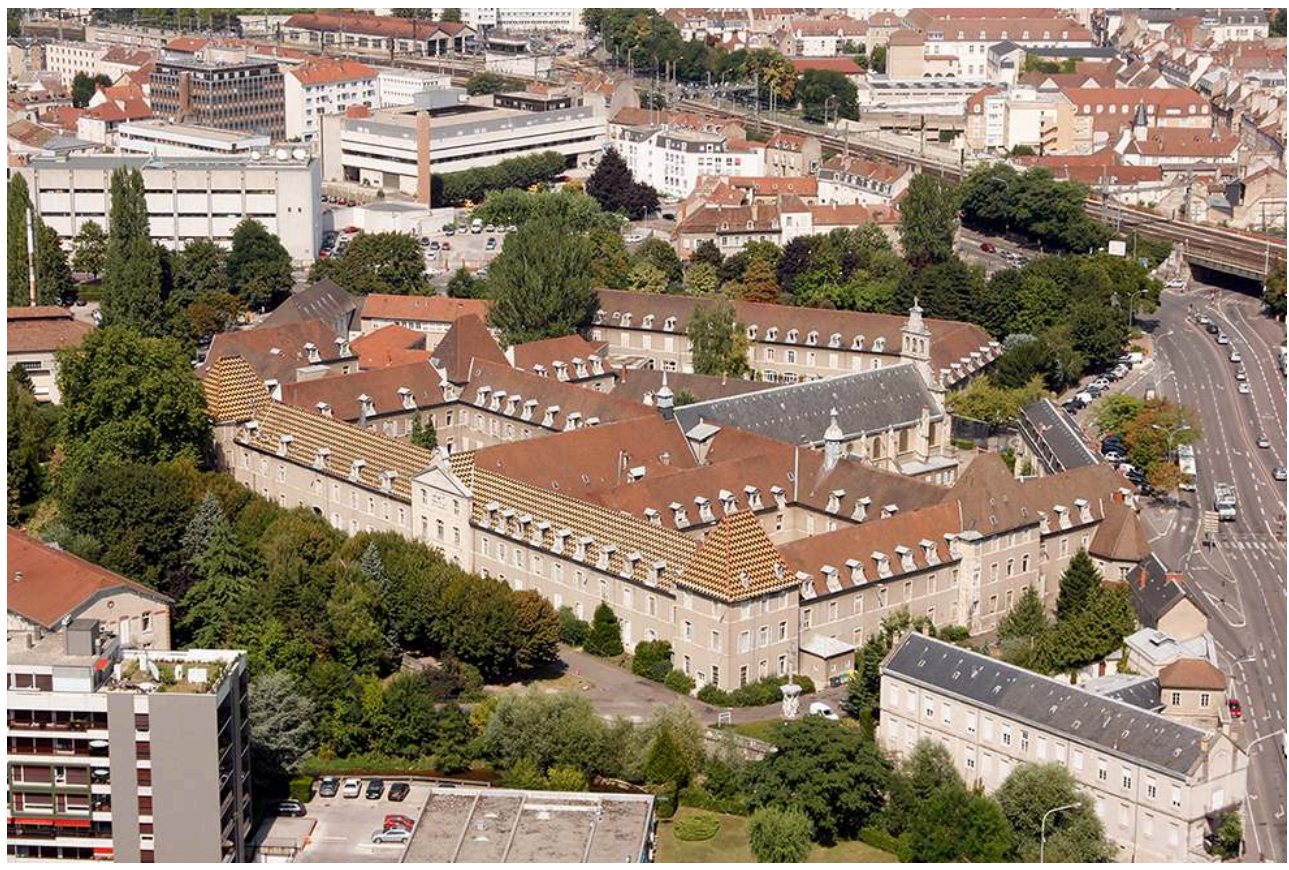

L'hôpital général de Dijon.

(c) Jean-Pierre Ramot/CHU Dijon Bourgogne.

2 Les différents services se répartissaient jusqu'au printemps 2015 sur trois sites principaux : l'hôpital général (fig. 1), site originel fondé en 1204 par Eudes III, duc de Bourgogne, le site du Bocage, inauguré en 1962, récemment rebaptisé "hôpital François-Mitterrand» (fig. 2) et le centre gériatrique de Champmaillot. Entre 2010 et 2015, l'ensemble des services installés dans l'hôpital général ont été transférés sur le site du Bocage. Prochainement, les bâtiments historiques, désaffectés, devraient être aliénés à la communauté urbaine du Grand Dijon qui a pour projet d'en faire une des quatre Cités internationales de la gastronomie (avec Tours, Rungis et Lyon) ${ }^{1}$.

Figure 2

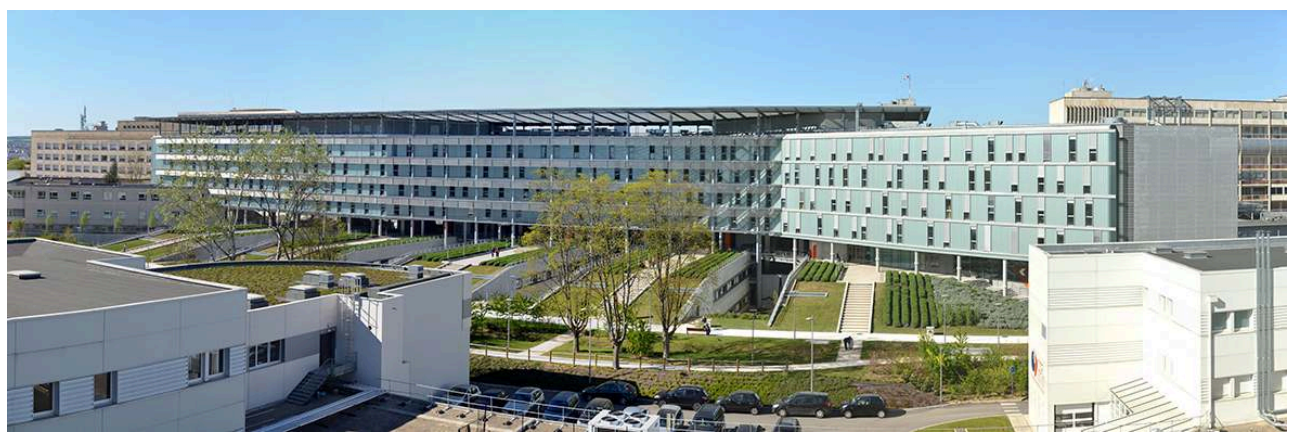

L'hôpital François-Mitterrand, anciennement dénommé hôpital du Bocage.

(c) Pierre Athias-TACTILL.

S'il fallait définir brièvement les collections patrimoniales du CHU Dijon Bourgogne, on pourrait identifier les objets «d'art et d'histoire» (tableaux, statues, meubles, argenterie), comprenant les objets du quotidien (couverts, assiettes, bouillottes ou bassins de lits et palettes à saignées) et médicaux (pots à pharmacie, clystères, balances, 
coffrets d'instruments de chirurgie) antérieurs au xxe siècle (fig. 3, fig. 4). Entre 2001 à 2006, grâce au partenariat entre l'agence régionale de l'hospitalisation (aujourd'hui agence régionale de santé), le CHU et le service régional de l'Inventaire général, un inventaire quasi exhaustif de ces collections a été réalisé par Bruno François au titre de ses fonctions de chargé de mission culture et patrimoine et de conservateur délégué des antiquités et objets d'art de Côte-d'Or avec la collaboration de Jean-Pierre Ramot, photographe du CHU. En cinq ans, 1925 objets ont ainsi été recensés sur les différents sites que compte l'établissement ${ }^{2}$. À la veille de la fermeture de son site historique, cet inventaire scientifique était une nécessité pour que l'hôpital puisse assurer une gestion raisonnée de ses objets patrimoniaux.

Figure 3

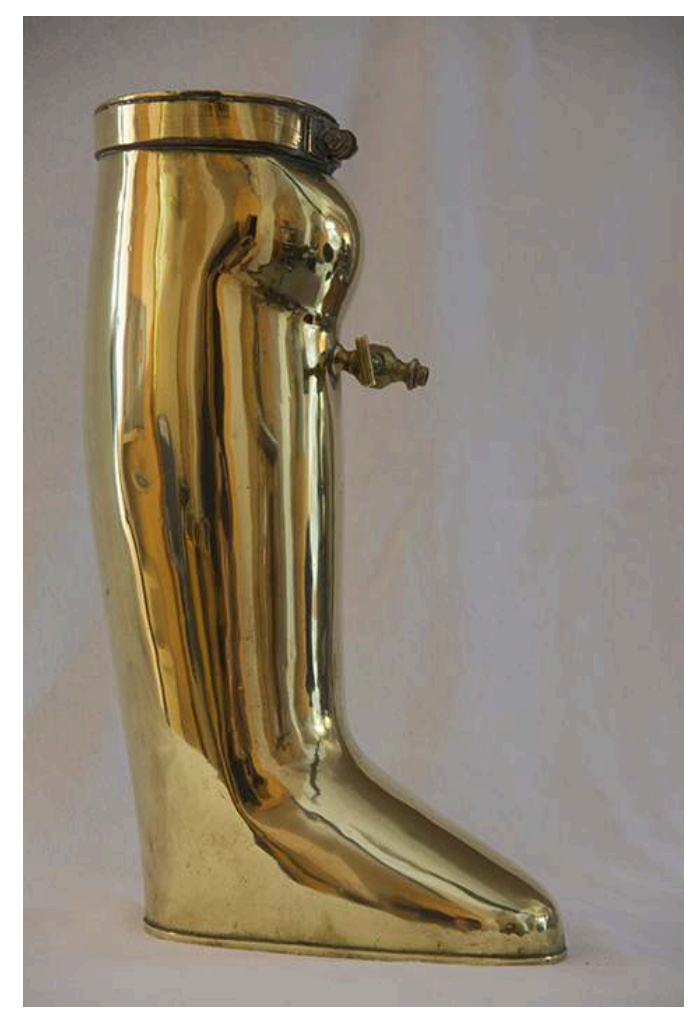

Botte médicale, selon le principe du docteur Junod, laiton, milieu du XIXe siècle, CHU Dijon Bourgogne. (c) Pierre Athias-TACTILL. 
Figure 4

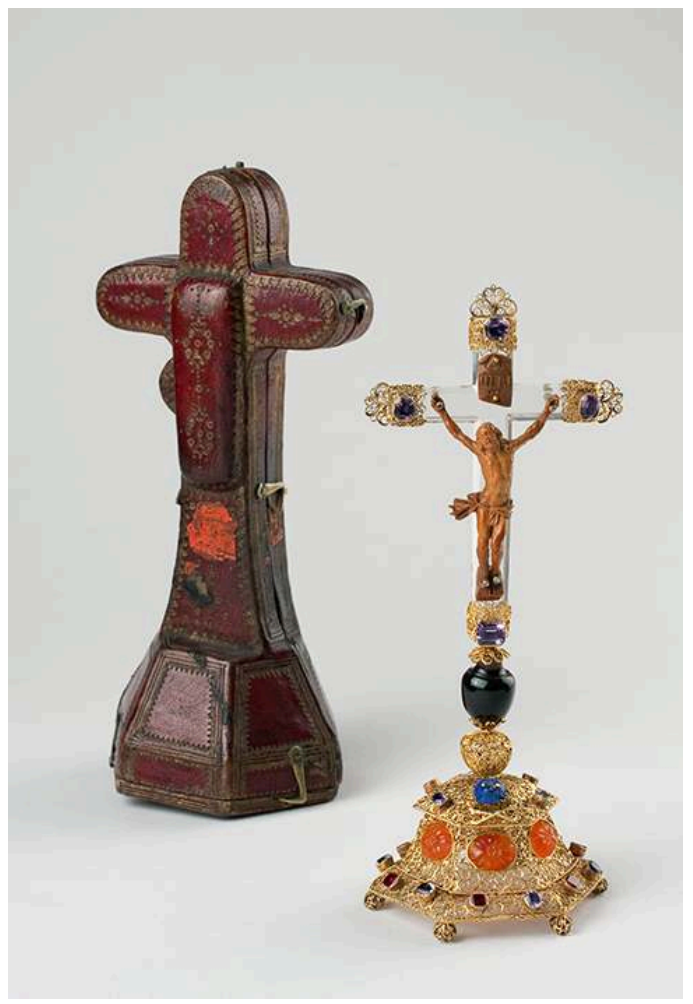

Croix dite de Christine de Suède, propriété du CHU Dijon Bourgogne en dépôt au musée de la Vie bourguignonne-Perrin de Puycousin, Dijon.

Phot. Perrodin, François. (c) CHU Dijon Bourgogne.

4 Cet important travail de connaissance a également permis, en 2007 et 2008, sur proposition de Bruno François, et avec l'autorisation du CHU, propriétaire, l'inscription au titre des monuments historiques de 299 objets dont 69 ont été classés en 2010. Ces mesures de protection sont venues s'ajouter aux 236 prises jusque-là.

Reflet de ses activités médicales de pointe et de ses activités de recherche, le CHU possède également une collection très importante d'objets «scientifiques et techniques" des $\mathrm{xx}^{\mathrm{e}}$ et $\mathrm{xxI}^{\mathrm{e}}$ siècles (instruments de chirurgie, matériel gynécoobstétrique, tubes à rayons $\mathrm{X}$, appareils d'anesthésie, pneumothorax artificiels, oscillomètres, échographes, toutes sortes de seringues, respirateurs...) récupérés dans les services, transmis par les membres du personnel et parfois même découverts dans les bennes destinées à la déchetterie. Ces 1659 objets inventoriés de 2010 à aujourd'hui, dont quelques-uns sont désormais protégés au titre des monuments historiques, témoignent de pratiques spécifiques de soins à l'échelle régionale. Toute la difficulté, pour les chargés des collections des centres hospitaliers, est de parvenir à comprendre l'objet scientifique conservé et à l'inventorier de manière à permettre sa compréhension par un tiers. L'utilisation d'un instrument est parfois propre à une spécialité médicale et si les informations ne sont pas collectées de façon précise au moment de la prise en charge de l'objet, son usage peut être perdu pour longtemps, voire pour toujours. À ce constat viennent s'ajouter des problématiques économique et logistique. En effet, avec la démultiplication des matériels, le manque de place et de financement pour les conserver de manière pérenne, des tris raisonnés doivent être faits au sein du CHU mais aussi dans les structures hospitalières et médico-sociales de la 
région pour ne garder que le matériel représentatif des activités de soin ou les objets rares. Afin de réfléchir aux critères à mettre en place et aux très nombreuses problématiques soulevées par la conservation d'objets scientifiques et techniques telles que la transmission des informations liées à l'usage, le fonctionnement, la dangerosité, la détérioration des matériaux dans le temps, un comité de sélection des objets $\mathrm{xx}^{\mathrm{e}}$ et XXI ${ }^{e}$ siècles (COSEL), composé de membres issus des services de soins, du service du biomédical et du monde muséal, a été créé au CHU Dijon Bourgogne en 2012. Ainsi, le but de ce comité ${ }^{3}$ est-il, d'une part de faire une sélection des objets proposés à partir de critères tels que les dimensions, le poids, l'état général, la possibilité ou non de le réutiliser à l'avenir, la dangerosité, les éventuels nettoyages indispensables pour rendre l'objet manipulable sans danger, l'ancienneté, la rareté, l'intérêt pour la compréhension des pratiques de soins par le public, etc. et d'autre part, de pallier (en partie) le manque de disponibilité du personnel soignant en constituant une mémoire des objets conservés. Ce comité a déjà permis, par exemple, de faire émerger le caractère exceptionnel d'un rein artificiel de marque Necker (fig. 5) utilisé au CHR (aujourd'hui CHU Dijon Bourgogne) au milieu des années 1950 et découvert en 2011 dans les caves du centre d'hémodialyse où il était mis en sûreté depuis plusieurs années pour lui éviter d'être volé (l'inox dont il est fait étant l'objet de convoitise). Ce rein a été inscrit au titre des monuments historiques en $2013^{4}$.

Figure 5

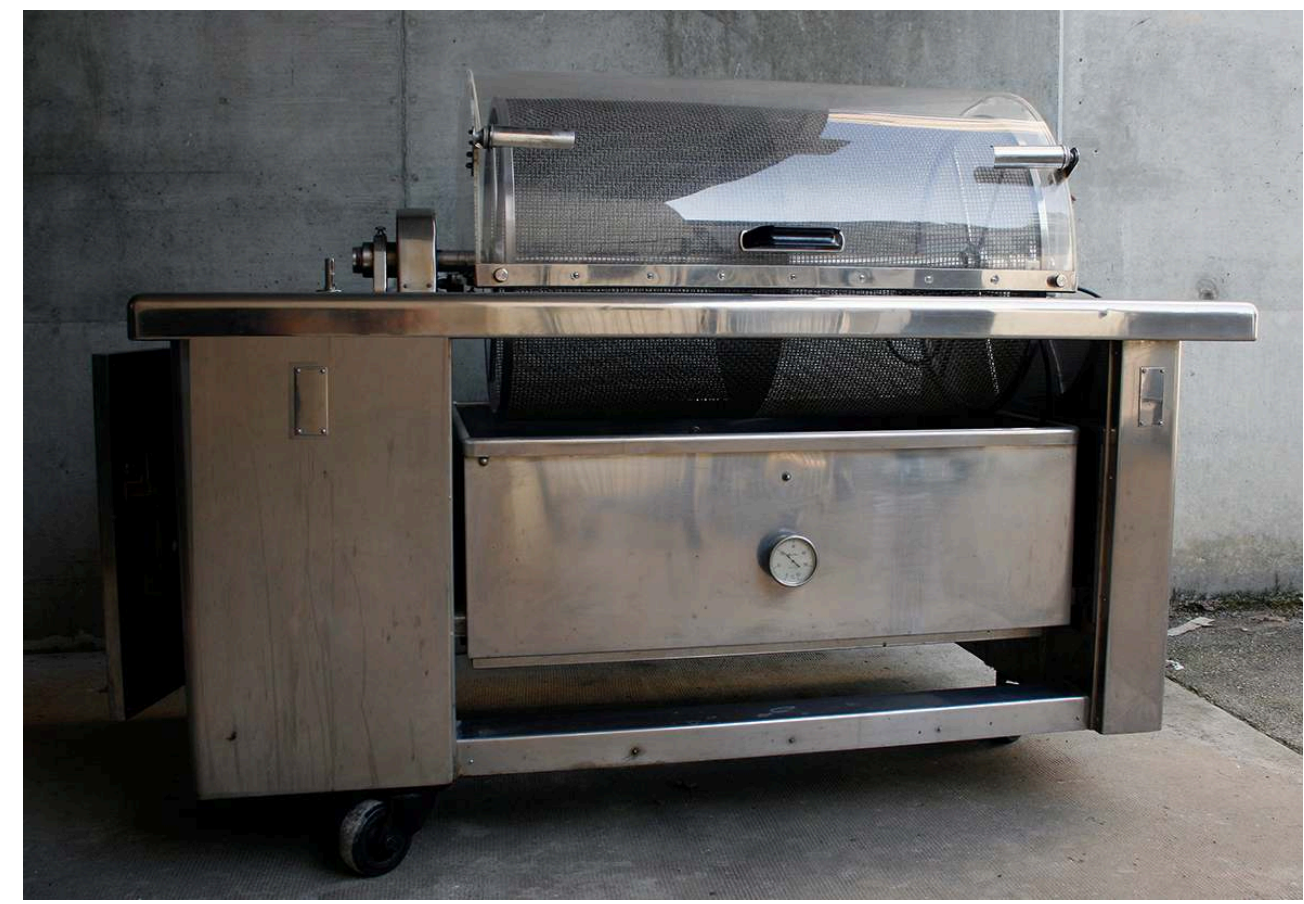

Rein artificiel de marque Necker, inox, verre, milieu des années 1950, CHU Dijon Bourgogne. (c) Jean-Pierre Ramot/CHU Dijon Bourgogne.

6 Ce comité, unique en France, a également été créé pour permettre le tri des matériels scientifiques et techniques hospitaliers à l'échelle régionale, à la suite des inventaires réalisés par Bruno François et moi-même pour l'agence régionale de santé BourgogneFranche-Comté. Le cadre officiel de ce comité permet d'attirer l'attention des structures hospitalières propriétaires sur ce patrimoine mal considéré, de mettre en 
lumière les spécificités anciennes ou actuelles des établissements et de sensibiliser l'intérêt du personnel pour les objets qui font partie de leur environnement quotidien. Enfin, les membres du comité, comme cela s'est produit pour le rein artificiel du CHU, peuvent signaler à la commission départementale des objets mobiliers (CDOM), qui n'a pas les compétences nécessaires pour évaluer ces thématiques récentes, des objets ou du matériel qu'il leur semble important de protéger au titre des monuments historiques, condition nécessaire pour assurer un statut juridique clairement défini aux objets souvent réformés et tenter d'éviter leur dispersion.

7 À la veille de la fermeture et de la cession de son site originel, le CHU Dijon Bourgogne a pris conscience de l'importance de conserver la mémoire de ces huit siècles d'activité de soin et de les faire connaître au plus grand nombre. C'est pourquoi l'établissement mène, depuis 2010, une politique très active de conservation et de valorisation de son patrimoine mobilier avec la création de $200 \mathrm{~m}^{2}$ de réserve sur le site FrançoisMitterrand. Pour permettre à tous de découvrir ses collections, 335 objets, dont la plupart sont protégés au titre des monuments historiques et proviennent des collections d'art et d'histoire, ont été mis en dépôt dans les musées dijonnais de la Vie bourguignonne-Perrin de Puycousin et d'Art sacré. Les objets de petites ou moyennes dimensions, en lien direct avec l'histoire de l'activité médicale, sont conservés et présentés dans des vitrines lors d'expositions thématiques renouvelées deux fois par an, accessibles dans le hall principal du nouvel hôpital. Pour les objets de grandes dimensions, certains sont conservés dans les réserves ou, lorsque leur état de conservation le permet, présentés dans des salles de réunion. Ils peuvent également être prêtés à d'autres structures ou associations pour des expositions ou tournages de films.

\section{NOTES}

1. - Voir le site: http://www.grand-dijon.fr/regards-sur/territoire-attractivite/cite-de-lagastronomie-et-du-vin/cite-internationale-de-la-gastronomie-51653.jsp [consulté le 05/01/2017]. 2. - Voir les dossiers en ligne dans la base Palissy du portail « architecture et patrimoine » du ministère de la Culture et de la Communication : http://www.culture.gouv.fr/culture/inventai/ patrimoine/.

3. - Sa composition varie en fonction des thématiques abordées: pneumologie, pharmacie, hémodialyse, etc.

4. - Cet appareil est une des premières machines à hémodialyse développée par la société Usifroid pour l'hôpital Necker à Paris au milieu des années 1950. Pesant près de $300 \mathrm{~kg}$, il était utilisé pour éliminer les déchets du sang par osmose, imitant ainsi l'action des reins. Le sang coulait à travers des tubes de cellophane enroulés autour du tambour central qui tourne dans un bain à température contrôlée contenant 100 litres de liquide de dialyse. Cette machine suit le principe élaboré en 1942 au Pays-Bas par Willem J. Kolff. 


\section{RÉSUMÉS}

À l'instar de nombreux hôpitaux, le CHU Dijon Bourgogne vient d'achever le déménagement de ses services de son site originel pour des locaux plus récents et donc plus fonctionnels. Riche des huit siècles d'existence de l'hôpital, le patrimoine mobilier de l'établissement est constitué d'objets "d'art et d'histoire ", comprenant aussi les ustensiles du quotidien et médicaux antérieurs au $\mathrm{xx}^{\mathrm{e}}$ siècle. Reflet de sa pratique médicale de pointe et de ses activités de recherche, le $\mathrm{CHU}$ possède également une collection très importante d'objets "scientifiques et techniques " des $\mathrm{Xx}^{\mathrm{e}}$ et $\mathrm{XxI}^{\mathrm{e}}$ siècles. Ces 1659 articles témoignent de méthodes spécifiques de soins à l'échelle régionale. Toute la difficulté est de parvenir à comprendre ces collections et à les inventorier pour les rendre accessibles au public. De plus, avec la démultiplication des matériels, le manque de place et de moyens financiers pour les conserver de manière pérenne, des tris raisonnés doivent être opérés au sein du CHU mais aussi dans les structures hospitalières et médicosociales. C'est pourquoi le CHU Dijon Bourgogne a choisi de se doter d'un comité de sélection des objets $\mathrm{XX}^{\mathrm{e}}$ et $\mathrm{XXI} \mathrm{e}^{\mathrm{e}}$ siècles.

Like many other hospitals, the Dijon Bourgogne university hospital has recently moved from its original buildings to others, more recent and more functional. The original hospital was eight centuries old and the movable heritage of the hospital comprises objects 'of art and history', ranging from everyday utensils to medical artefacts dating from before the twentieth century. Bearing witness to the place's advanced medical practices and its research programmes, the hospital also holds a significant collection of 'scientific and technical' objects from the twentieth and twenty-first centuries. The 1,659 objects bear witness to the specific methods of healing at the regional level. The difficulty lies in understanding these collections and inventorying them in order to share them with the public. Wit the increase in the number of medial appliances, the lack of space and of financing to keep these objects permanently, reasoned selection processes have to be undertaken both within the university hospital and in other hospital and medicosocial structures. The CHU of Dijon-Bourgogne has set up a special committee for this task of selecting the pieces to be kept from the twentieth and twenty-first century objects.

\section{INDEX}

Mots-clés : Dijon, Bourgogne, CHU, hôpital général, inventaire du patrimoine mobilier, objets scientifiques et techniques, comité de sélection, XXe siècle, XXIe siècle

Keywords : Dijon, Burgundy, university hospital, general hospital, inventory of movable objects, scientific and technical objects, selection committee, twentieth century, twenty-first century

\section{AUTEUR}

\section{ADELINE RIVIÈRE}

Chargée des collections patrimoniales, CHU Dijon Bourgogne, chargée d'inventaire et de valorisation du patrimoine, agence régionale de santé Bourgogne-Franche-Comté, conservatrice déléguée des antiquités et objets d'art de la Côte-d'Or adeline.riviere@chu-dijon.fr 